\title{
5 APPLYING USAGE MODELS TO INNOVATE INFORMATION TECHNOLOGY SOLUTIONS
}

\author{
Sigal Louchheim \\ Intel Corporation \\ Folsom, CA U.S.A. \\ Petra Langwald \\ Judy Ossello \\ Intel Corporation \\ Hillsboro, OR U.S.A.
}

\begin{abstract}
The pro-innovation bias is frequently reflected in the deployment of new information technologies by IT departments. The implicit assumption is that IT innovations should be adopted, and if they are not, the problem lies with the target audience rather than with the innovation itself or with the diffusion methods applied.

In this work, we have taken a usage model driven approach to identify innovative opportunities for improving collaboration within geographically dispersed teams of design engineers. Our primary goal was to establish the basis of a repeatable process using this approach in IT, driving the development of innovations that are desirable, useful, and usable to the target audience.

A usage model describes users and their goals, as well as the context and process of system use. Our process focused on creating three model components: ethnographic data, personas, and scenarios. From these components, we identified short-, mid-, and long-term opportunities for improving the collaboration experience.
\end{abstract}

Keywords IT innovation practices, IT adoption, user-centered innovation, usage model

Please use the following format when citing this chapter:

Louchheim, S., Langwald, P., and Ossello, J., 2008, in IFIP International Federation for Information Processing, Volume 287, Open IT-Based Innovation: Moving Towards Cooperative IT Transfer and Knowledge Diffusion, eds. León, G., Bernardos, A., Casar, J., Kautz, K., and DeGross, J. (Boston: Springer), pp. 83-96. 


\section{INTRODUCTION}

Employees at large information technology companies, such as Intel Corporation, are accustomed to deployments of newer and faster technology and products with more features. We usually justify these deployments from business or technology perspectives, such as decreasing total cost of ownership, increasing security, or enabling new business opportunities. As Rogers (2003) discusses, the agency that sponsors the innovation has a pro-innovation bias. Rogers describes the pro-innovation bias as the implication that the innovation in question is diffused and adopted by the entire target audience, quickly, without resistance and with no changes made to the innovation.

End users often do not have a voice in this process. Generally, however, users want to accomplish a goal or they desire an experience, and the technology is a tool to achieve these needs rather than an end in itself, which can create challenges in the diffusion of the information technology product. To build desirable, useful, and usable products, we need to understand who uses the products and what their needs are. This understanding constitutes the usage perspective. The other two perspectives that any product or service has are the technology and business perspectives. The balanced integration of all three perspectives yields the most compelling solutions as explained by Simmons (2006) and portrayed in Figure 1.

When Brown (2002, p. 107) details the learnings of Xerox PARC, he explains that

the successful company of the future must understand how people really work and how technology can help them work more effectively....It must rethink traditional business assumptions and tap needs that customers don't even know they have yet.

He states that researching new work practices is equally important to researching new technologies and details that

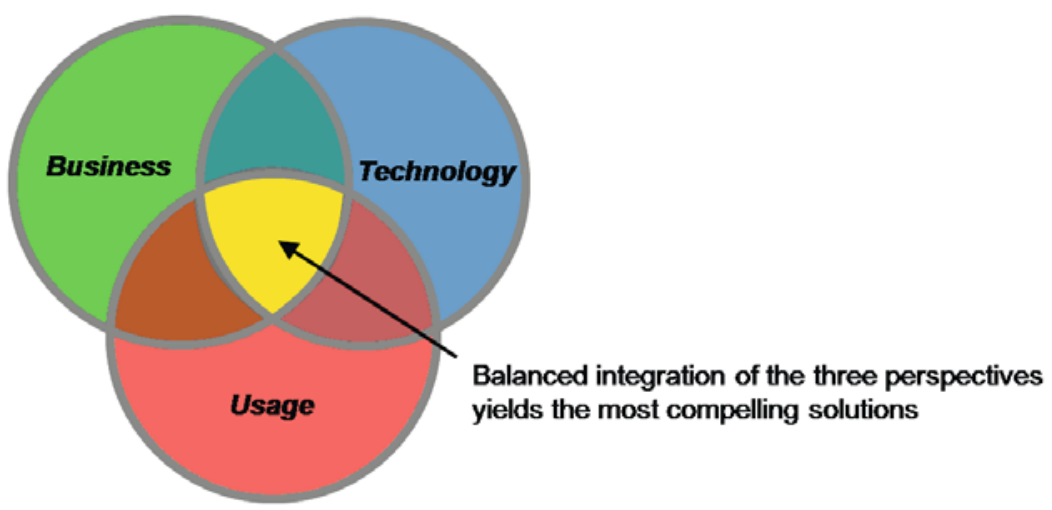

Figure 1. The Three Perspectives: Usage, Business, and Technology 
this means going beyond the typical view of technology as an artifact — hardware and software - to explore its potential for creating new and more effective ways of working, what we call studying technology in use.

We address this very challenge in our work, by developing a usage model driven approach. This approach enhances the way we create and innovate by deriving the requirements from user needs and goals.

A challenge was presented to our IT department: engineering team managers know that their geographically dispersed design engineers faced difficulties collaborating, but were not sure of the best way to address the problems. We suggested a usage model driven approach to discover opportunities for improving the engineers' experience. We have not traditionally used this approach for IT solutions.

This work has two goals: to establish the basis for a repeatable usage model driven approach and apply it on a case study. Our case study is on improving the globally dispersed collaboration experience of design engineers. Hence, this work describes two results: (1) The usage model driven approach we defined. This is a repeatable process that that can be used in future innovative projects in IT and make their adoption more likely. (2) The high-impact opportunities for business units and IT to improve the collaboration experience among dispersed design engineers.

The rest of the paper is structured as follows. In section 2 we briefly describe the usage model structure, followed by an overview of the usage model driven approach we introduce in section 3. In the following four sections we describe each one of the components of the usage model driven approach: ethnographic data in section 4, personas in section 5, scenarios in section 6 and identifying innovation opportunities in section 7 . In section 8 we conclude with a discussion.

\section{THE USAGE MODEL}

To provide a compelling user experience, we must identify who our users are and understand their goals, needs, and desires in context. This means also understanding our users' social and physical environment.

We apply a usage model driven approach to achieve this goal. This approach can be valuable during the early stages of project definition to explore the viability of an innovation. The usage information that we gather at this early stage feeds into subsequent planning and development, helping guide decisions in terms of the user experience and helping us create solutions that meet user goals. The results can also be applied in later phases of the project, all the way through the validation of the solution and the application of the diffusion channels.

\subsection{Usage Model Structure}

A usage model, defined by Simmons (2006), captures data about users and their goals, the context in which they use the system, and the way they use it. Usage models describe system usage in context, at a level that identifies the system's benefits to its users. 


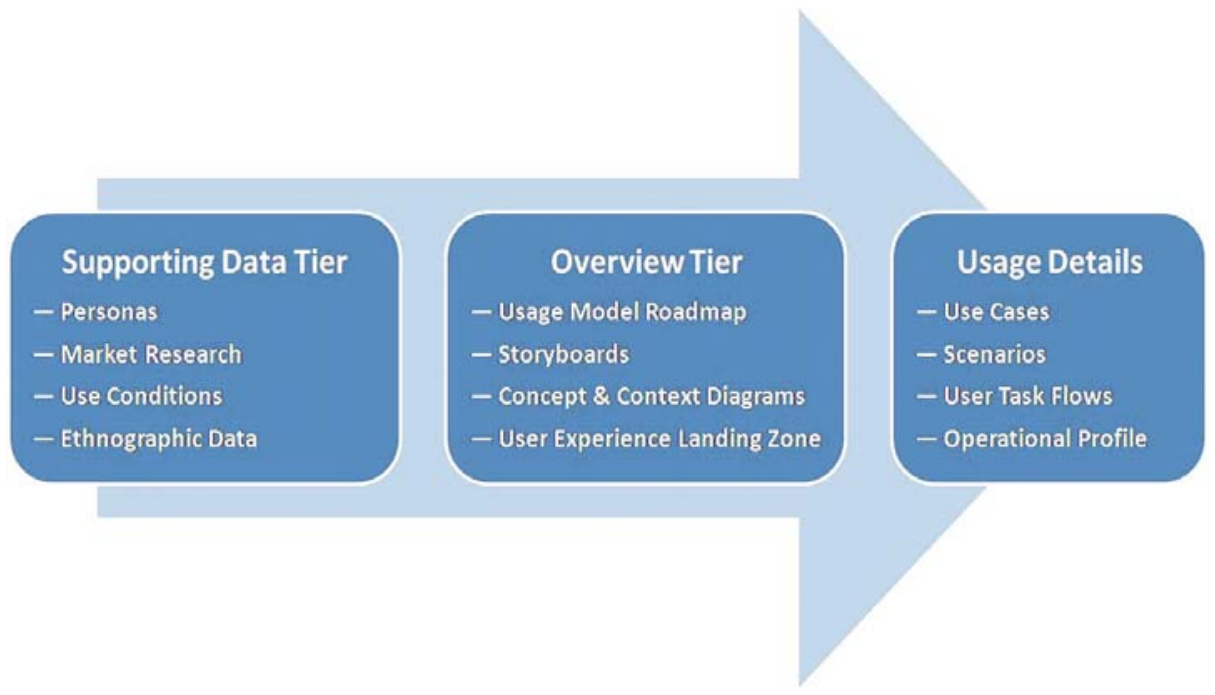

Figure 2. Usage Model Tiers and Components

The usage model driven approach consists of three steps.

- User: identify the innovation's (system's) users and discover their goals

- Usage: define how the users use the system

- Requirements: derive the functional and nonfunctional requirements, transforming the description from a user's perspective into a technical description of the innovation

As depicted in Figure 2, the usage model structure contains three tiers, corresponding roughly to the three steps above: supporting data, usage overview, and usage details.

As Simmons (2005) explains, the supporting data tier provides the depth and background to the other two tiers in the model. It includes quantitative data based on market research and qualitative data emerging from ethnographic studies. For a specific project or innovation, we develop selected components of the model depending on the scope and context of the project. The usage overview tier forms the introduction to the information in the other tiers, and is especially valuable to management because it summarizes findings, whereas the other two tiers provide details. The usage details tier includes the main descriptions of the system use. This information guides the design, validation, and communication of the project, as well as helps manage the scope by relating it to usage. This tier plays a key role in tracing the system's technology features and their impact on users. For example, we could understand the impact that removing a feature might have on the user experience by tracing back the requirement using the usage details. A full description of the usage model is provided by Simmons (2006). 


\section{OVERVIEW OF THE USAGE MODEL DRIVEN APPROACH}

We had dual goals in this work: first, to define the basis of a repeatable process that we could use in future innovative projects in IT, both in their formation as well as in their successful diffusion; second, to discover high-impact opportunities for business units and IT to improve the collaboration experience among globally dispersed design engineers, which was the challenge that was presented to us.

To achieve these goals, we initiated a project aimed at taking a fundamentally user-centered approach to gain a better understanding of design engineers' dispersed collaboration needs. Through this work we developed the repeatable approach that enables us to focus on user-derived opportunities for innovation that we describe here.

We knew that there were gaps in our understanding of design engineers. To address that, we focused our efforts on developing research components that could improve our understanding, rather than imposing a solution that would be a fit for our own collaboration needs. We developed three usage model components: ethnographic data and personas in the supporting data tier and scenarios in the usage details tier, as shown in Figure 3. The personas enable us to clarify and understand who the target audience is and what the target audience's goals and contexts are, as detailed in section 5. The scenarios enable us to define how the system is used and how it will be used in terms that relate directly to the target audience's goals.

The ethnographic data we collected through real-world observations and interviews guided our development of both the personas, which describe who engineers are, and the scenarios, which describe how they act in their contexts to achieve their goals. We also had access to limited market research data that helped us frame the scope of the ethnographic data collection; we chose three or four people to observe and interview in each of the audience segments.

One way of identifying opportunities or getting user requirements is to approach the users, or the customers, and ask them to identify their needs. However, users may have difficulties in articulating their needs on demand. For example, many of the managers of globally dispersed design engineers are familiar with some of the issues contributing

Collect and Analyze Ethnographic Data
Create Personas

\section{Define Scenarios}

Identify

Opportunities

Figure 3. The Usage Model Driven Approach Overview 
to poor collaboration, such as the ones imposed by technological and physical constraints. However, these managers may have difficulty in articulating how team culture and improvised temporary solutions (work-arounds) impact collaboration. As Spradley (1979) notes, our cultures provide us with the lenses through which we see, interpret, and describe the world. Users are similarly "imprisoned" in "a particular reality that is taken for granted as "the reality"' (p. 10) and should not be expected to be able to articulate, on demand, their needs. The process usage model driven approach we introduce in this work allows us to identify the user needs - including the latent user needs - and derive from them the opportunities to improve their experience.

The following four sections describe the four components of our usage model driven approach, as well as key learning we have gained. Throughout our work, we have found that since the usage model approach is relatively new to our IT department, few people had experience with it. Gaining proficiency in usage models is important for the seamless implementation of this process. It was also important to understand the constraints that confine the process: the complexity of the proposed innovation, the resources available to perform the data collection and analysis, the time lines, etc.

\section{ETHNOGRAPHIC DATA}

According to Spradley (1979, p. 5), "the essential core of ethnography is this concern with the meaning of actions and events to the people we seek to understand," making ethnographic data essential in creating personas. He explains that there are three general sources for cultural inferences: what people say, how they act, and the artifacts they use. Ethnography seeks to capture an understanding of human culture that includes both the explicit and tacit knowledge with which members of the culture are familiar, without presupposing any hypotheses, or, as Spradley (1979, p. 4) says, "ethnography starts with a conscious attitude of almost complete ignorance" and that "rather than studying people, ethnography means learning from people."

\subsection{Ethnographic Data Collection}

To gather ethnographic data, we observed and interviewed 22 design engineers working on silicon and board design projects at four different sites in the United States, India, and Malaysia. Spradley $(1979,1980)$ describes observation and interview methodologies.

To identify potential participants to observe and interview, we started by identifying four major silicon and board design projects with globally dispersed teams across several locations. The projects were in different product life cycle phases such as exploration, planning, development, and deployment. Two main variables influenced our individual recruitment efforts from each team: project role and physical location. To focus on highly collaborative work responsibilities we looked for individuals who play a part in consolidating work products across several roles. These individuals do not necessarily have management authority, but may be responsible for project deliverables. 
We recruited potential participants through their managers; we first contacted the managers for approval to observe and interview engineers within their organizations. The managers then solicited volunteers. We found it best to contact these participants directly to introduce the project and the methodology. Since the observations and interviews require a time commitment from the users and their management's support, it is important to contact both. The process of identifying appropriate participants and getting a commitment both from them and their managers took us approximately three weeks. Some potential participants perceive observations and interviews as intrusive. It was important to detail how the participants' privacy would be protected and clarify in advance what information and activities will be collected and observed. We note that the population sample we observed and interviewed were a self selecting sample, comprised of volunteers.

We observed participants for an average of two half-days, followed by an interview. To observe participants, members of our team unobtrusively shadowed participants during their work days as they worked at their desks, attended meetings, and performed other activities. It is important for the observations to include both the person being observed, what they say, and their activities, as well as their environment, both physical and the people with whom they interact. We took comprehensive notes throughout the process. We believe that photographs, video, and audio recordings could also help minimize the risk of losing information.

During the open-ended, semi-structured interviews, we asked participants detailed questions about their backgrounds, the processes and tools they used to do their jobs, with whom they typically collaborated, challenges they experienced in collaborating, and other areas.

\subsection{Ethnographic Data Analysis}

We stored all this data in a central repository, and developed our results by iteratively comparing data gathered during the observations and interviews of different participants and looking for patterns in the similarities and differences between them. Analyzing these patterns helped us to organize participants' actions and motivations in common situations into eight to ten general areas of interest, or themes. We relied on this analysis during the rest of our usage model development.

We have found that it was valuable to have two people conducting the observations and the interviews. When two people conduct the interview, one is able to focus on guiding the interview and the other on taking notes. Both in conducting interviews and in observation, when two people conduct the process, they can discuss the data and remind each other of salient points that the other might have missed or forgotten, and lead to a faster and more thorough discovery of significant patterns in the data.

It is important to allocate enough time for data analysis, creating the model components, and iterative validation of results. For every hour we spent on observations and interviews, we spent about three hours analyzing the data and writing the other usage model components. 


\section{PERSONAS}

Cooper (2004) and Cooper and Reimann (2003) introduced personas to develop a precise description of a narrow target audience and what they wish to accomplish in context. Personas are hypothetical user archetypes of actual users. They are defined with rigor and precision, based on real data; ethnographic data is one of the primary sources of data for persona development, the other being market research. Personas are believable: they have a name, a face, personal details, and so forth. This allows people who use them to create a mental connection to the persona described, even though it does not describe a real person.

Personas are differentiated by their goals, their needs, their desires, and their contexts. They are described within their social, physical, and environmental contexts and may include workflow and skill levels as well as quotes to make the persona more credible and complete. Personas are relevant to the market, as shown through quantitative research. The personas enable decisions to be made based on user goals, and reduce the chance we design for ourselves or people just like us or that we include features in the innovation or project simply because they are appealing to us. Personas ensure that the entire project team, including the stakeholders, has a shared vision and reduce the chance we will make "convenient" assumptions about users and their lives.

Personas help focus on meeting user goals. Personas have five major characteristics: (1) they are memorable; (2) they are based on real data but are not a description of a real person, rather are a deliberate composite that only includes deliberate quirkiness; (3) they are narrowly defined; (4) they are not an average of the data, but rather highlight specific target audiences on which we are focusing; (5) they are representative, capturing key characteristics of the target audience segment.

We began the persona development process using a template that included the persona's goals, attitudes, skill levels, challenges, and frustrations as well as a detailed narrative of the persona's environment. As we established our personas, we iterated through periods of data analysis, persona writing, and reviews. During the data analysis, we examined the patterns and themes emerging from the ethnographic data, identified recurrent themes, and grouped them by similarities. This helped us identify common collaboration goals, challenges, and activities around which we built the personas. We then filled in this structure with narrative descriptions based on the ethnographic data typical of each persona. Note that we did not include suggestions on product design.

We found that it is critical for those involved in conducting observations and interviews to also take part in analyzing the data and creating personas and scenarios. Their familiarity with the details of the data brought richness to the final results. Similarly, prior experience and expertise in creating personas and scenarios clearly enhances the results.

Through this process, we identified four personas, each with a different collaboration focus based on how the individual's work connected to that of others.

One of our personas described a design engineer technical lead, whom we named Pradeep Mishra. He coordinates the work of his team on subsections of chip design, takes the lead in reviewing architectural specifications and changes, and collaborates with validation and mask design teams during the design process. This approximately 900-word persona describes his other work responsibilities and skills in detail. It also 
provides personal information that helps build a more complete picture, such as his age, education, the languages he speaks, and how long it takes him to commute to his work site in India. The persona describes his frustrations, such as sometimes being unable to reach team members to troubleshoot problems. Very importantly, it also describes his goals, including fast access to information and people to solve the team's issues. In section 6, a sample scenario describing Pradeep problem solving across sites is described.

Our other personas described a project manager and two engineers, each with a different collaboration focus.

Note that we developed personas around our user population, those design engineers whose dispersed collaboration experience we were challenged with improving through our proposed innovations. In our analysis we did not include those people who will be paying for the diffusion and implementation of the innovation, but will not necessarily be using it. Their needs may need to be included, and in some cases, even personas may need to be developed for them, but that will need to be done separately. Similarly, we excluded the support personnel, stakeholders, and subject matter experts and did not create personas for those classes, either.

\section{SCENARIOS}

A scenario is a narrative that describes how a user interacts with the innovation, which can be a product or a service, to achieve a goal or task. A scenario is a realistic depiction of that interaction, and like personas, scenarios are based on supporting data and are described in context. Scenarios can build on personas, using a persona in the central role. A scenario typically describes a single path of interaction between the persona - including potentially other protagonists - and a product or service. A single path refers to the specific chain of activities with which the persona is engaged given the options available at the time, without including "what-if" conditions that would describe additional, divergent paths that could have been taken under different conditions. Alexander and Maiden (2004) provide a comprehensive range of scenario techniques and discuss the practices in this area both from the academia and the industry perspectives.

We used the scenarios to derive opportunities and requirements and to communicate with stakeholders.

Scenarios are very effective when they present richly detailed descriptions. To develop our scenarios, we reviewed our personas and ethnographic data and identified tasks necessary for the personas to meet their collaboration goals.

For each persona, we identified two to four scenarios. To help us understand current tasks and difficulties, we created an as-is state of the scenario describing current conditions. Based on the persona's goals and needs as well as the challenges of the existing environment, we derived a to-be state describing the persona's desired state. We made to-be scenario states technology agnostic to avoid imposing design solutions at an early stage.

Scenarios covered specialized and general situations such as meetings, integrating versions of design files, tracking issues, and interpreting new specifications. We present a sample scenario below that focuses on problem solving across sites. The persona this scenario is focused on is Pradeep Mishra, a design engineer technical lead. We first 
present the as-is state, describing the current condition in real-time problem solving and design interaction. Then, we present the to-be state, describing in a technology-agnostic manner the state where the effect is of improved effectiveness of communication. Notice that the to-be scenario is technology agnostic, so that no technology requirements are imposed at this early stage. The gap analysis between as-is and to-be versions of scenarios enables the identification of opportunities to innovate and improve the collaboration experience.

\subsection{As-Is Sample Scenario}

Pradeep and his team of design engineers in India are preparing for a phone meeting with the validation engineers in Russia. Pradeep called the meeting because the teams cannot understand why validation tests are failing, despite exchanging e-mails about the problem for two days. He usually prefers e-mail and instant messaging for these discussions: phone meetings are difficult because each team's accent is almost incomprehensible to the other. However, in this case, the e-mail exchanges are too slow and the teams are not making progress.

The two teams call in to the meeting. Pradeep asks if the Russian group has the presentation file he sent earlier-he cannot share it using real-time collaboration software because of the unstable network connection between the two sites. The Russian team leader says he is projecting the presentation in their conference room. The file is large, containing text, code, and drawings; it took Pradeep two hours to put together because he tried to anticipate everything they might discuss during the meeting.

Pradeep talks through the slides. As long as he stays with the prepared material, the Russians respond with thoughtful questions that make Pradeep believe they understand what he is saying.

However, he wishes he could see if they were confused or nodding in agreement. When one Russian, Ivan, describes a logic flow, Pradeep's team shakes their heads, so Pradeep asks Ivan to repeat himself so they can understand. Frustrated, Pradeep eventually asks an engineer on his team, Anand, to draw what Ivan is describing. Pradeep's team cannot understand Anand's sketch. Pradeep finally asks Ivan if he can make a sketch and send it to him later so the team can look it over.

\subsection{To-Be Sample Scenario}

Pradeep's team has been corresponding with the Russian group for two days but they still cannot understand why validation tests are failing. To solve the issue quickly, Pradeep schedules a virtual meeting in which the participants can see visual cues from each site.

Pradeep discusses why his team is experiencing difficulties. He can see that Sergei, one of the Russian engineers, is looking tired, but the rest of the Russian team is looking alert. Anand, on Pradeep's team, can tell that Ivan understands the point he made, and the conversation moves quickly. Once, Ivan asks Pradeep to repeat a phrase three times. Ivan still cannot understand, so Pradeep communicates using a written message. Every- 
body in India can see the Russian engineers nodding when they see it. When Ivan discusses the logic flow problem, another engineer creates a visual sketch for the group. The teams agree that this is a key problem, so Pradeep assigns a team member to work with Ivan to solve it, and the meeting ends early.

\section{IDENTIFYING OPPORTUNITIES FOR INNOVATION}

By following the usage model driven approach we have defined, we were able to identify the goals and needs as well as the existing needs and challenges in collaboration of globally dispersed engineers. To discover high-impact opportunities for innovation that will improve the collaboration experience of geographically dispersed design engineers, we identified the gaps between the existing state and user goals. We exposed these gaps by comparing the as-is and to-be versions of the scenarios that we developed for the design engineers.

These gaps revealed the frustrations design engineers encounter in performing their tasks, the hurdles that prevent them from performing their tasks efficiently, and opportunities for improving their dispersed collaboration experience.

The usage model driven approach revealed short-, medium-, and long-term opportunities for innovation, which are differentiated and enumerated below. We validated these opportunities with user representatives including the engineers themselves, our subject matter experts, stakeholders, and our sponsors. Together, we prioritized the innovation opportunities and recommended which should be pursued first to meet the business unit's needs.

\subsection{Short-Term Opportunities}

Short-term opportunities are those where the problem domain and potential solutions are well understood. The innovations in this case have been developed and are ready to be tested through proof of concepts and pilots as part of the initial diffusion into the target audience. The understanding of the target audience that we have accumulated through this process will continue to play a role through this process.

The short-term opportunities that we have identified through this work include

- $\quad$ Seeing nonverbal cues during the dispersed collaborative interaction. This is particularly important in Asia, where even more attention is paid to nonverbal cues.

- $\quad$ Sharing visual artifacts, such as sketches, across sites.

\subsection{Mid-Term Opportunities}

Mid-term opportunities are those where the problem domain is well understood but the solution domain is not. That means that we need to explore potential solutions before selecting one for testing and then diffusion. 
The mid-term opportunities we have identified through this work include

- Decreasing delays in sharing information between sites. Specific focus areas that we identified under this theme include

- Communicating decisions and plans

- Increasing availability and accessibility of information

- Ensuring information shared locally is communicated globally to the team

- $\quad$ Sharing sketches or large files

- Facilitating an environment that supports synchronous, asynchronous, and external collaboration.

- Finding people and managing interruptions. For example, in India we observed frequent interruptions in meetings due to participants answering phone calls.

\subsection{Long-Term Opportunities}

Long-term opportunities are those where we need to investigate the problem domain further to fully understand it before we can explore the solution. These opportunities were not directly stated in the data gathered through observations and interviews, but surfaced when we analyzed the data. An example for a long-term opportunity is the lack of consistent and comprehensive support of the engineering methodology within the system architecture.

\section{SUMMARY AND DISCUSSION}

Christensen (2005) highlights the role of innovation for sustained success of companies. Diffusion of innovation remains a significant problem that confronts IT organizations. The evolving and rapidly changing environment that IT needs to support requires a keen understanding on the part of IT practitioners of not only the technology and business perspectives, but also of the usage perspective. The repeatable process that we developed can enable other IT teams to achieve that: to satisfy — and even delight - their users by utilizing the understanding they gain of who their users are, and of their users' goals, needs, challenges, frustrations, and environments. In short, by applying the repeatable process we have described, teams can gain an understanding of the social and physical contexts of their target audience and how their target audience is going to interact with the proposed innovation, how it will be used.

The information on both the users in their context and how they will use the innovation can be used to derive requirements for an innovation that will be easier to diffuse, since it will answer the critical question of "what's in it for me" for users in terms that incentivize their adoption of the innovation. As Rogers (2003) discusses, this is a significant challenge in diffusion of innovation in general, and it is true for us in IT.

Increasing productivity remains a central challenge for IT, and as organizations become globally dispersed, the challenges of working in those environments become significant for IT.

In this work we tackled both issues. We examine how to create a repeatable process through which IT innovations could be more oriented to addressing user goals. We also 
address the productivity challenge by examining how to innovate and improve the globally dispersed collaboration. We found that the usage model driven approach provided an effective way to explore problems in collaboration among dispersed project teams. The approach yielded valuable results in a situation where we knew difficulties existed but were not sure of the best way to address them.

By applying this approach at an early stage, we identified short-, medium-, and longterm opportunities for improving collaboration. We also developed the basis of a repeatable process for use in IT projects.

Deploying user-centered methods within IT presents some specific challenges. Users and IT may have conflicting goals and motivations, solutions are often based on off-the-shelf solutions from vendors, and IT may prefer one-size-fits-all solutions while end users prefer solutions that solve their unique problems. We believe that our usage model driven approach addressed these challenges by helping better understand users' goals. By applying the lessons we learned during this project, we expect to improve the innovation process for future IT projects.

Employing a usage model driven approach is a change in the IT development process, which creates another innovation diffusion challenge: overcoming inherent resistance of the IT organization to change, even when the change promises to deliver benefits. It will require commitment from IT and its business unit partners to understand and address business unit needs using this approach, and is the setting for our future work.

\section{References}

Alexander, I. F., and Maiden, N. 2004. Scenarios, Stories, Use Cases Through the Systems Development Life Cycle, Chichester, England: John Wiley \& Sons.

Brown, J. S. 2002. "Research That Reinvents the Corporation," Harvard Business Review (80:8), pp. 105-115.

Christensen, C. M. 2005. The Innovator's Dilemma: When New Technologies Cause Great Firms to Fail, Boston: Harvard Business School Press.

Cooper, A. 2004. The Inmates Are Running the Asylum: Why High Tech Products Drive Us Crazy and How to Restore the Sanity (2 ${ }^{\text {nd }}$ ed.), Indianapolis, IN: Sams Publishing.

Cooper, A., and Reimann, R. 2003. About Face 2.0: The Essentials of Interaction Design, Indianapolis, IN: Wiley Publishing Inc.

Rogers, E. M. 2003. Diffusion of Innovations ( $5^{\text {th }}$ ed.), New York: Free Press.

Simmons, E. 2005. "The Usage Model: A Structure for Richly Describing Product Usage During Design and Development," in Proceedings of the $13^{\text {th }}$ IEEE International Conference on Requirements Engineering, Paris, France, August 29-September 2, pp. 403-410.

Simmons, E. 2006. "The Usage Model: Describing Product Usage during Design and Development," IEEE Software (23:3), pp. 34-41.

Spradley, J. P. 1979. The Ethnographic Interview, New York: Holt, Rinehart and Winston. Spradley, J. P. 1980. Participant Observation, New York: Holt, Rinehart and Winston.

\section{About the Authors}

Sigal Louchheim is an internationally recognized expert in the area of interestingness, the discovery of what is interesting (new, actionable, etc). She received her Ph.D. in Computer 
Science in 2003. Sigal is focusing on creating compelling user experiences and adoption strategy and design. Sigal can be reached at sigal.louchheim@intel.com.

Over the past 16 years Petra Langwald has program managed complex projects and aligned activities across international teams in various disciplines: software, marketing, hardware, and IT. She has worked in technology companies in Germany, France, and the United States as a program manager and business analyst. Petra can be reached at petra.langwald@intel.com.

Judy Ossello studied the adoption and development of information technology-based solutions that solve strategic and tactical business problems at DePaul University. In addition to working for Intel, she is pursuing an MBA with Babson College to develop a greater understanding of information technology-based solutions from a managerial, financial, and organizational perspective. Judy can be reached at judy.ossello@intel.com. 